\title{
Nodal Solutions for Problems with Mean Curvature Operator in Minkowski Space with Nonlinearity Jumping Only at the Origin
}

\author{
Wenguo Shen \\ Department of Basic Courses, Lanzhou Institute of Technology, Lanzhou 730050, China \\ Correspondence should be addressed to Wenguo Shen; shenwg369@163.com
}

Received 14 December 2019; Accepted 4 February 2020; Published 13 April 2020

Academic Editor: Richard I. Avery

Copyright (c) 2020 Wenguo Shen. This is an open access article distributed under the Creative Commons Attribution License, which permits unrestricted use, distribution, and reproduction in any medium, provided the original work is properly cited.

In this paper, we establish a unilateral global bifurcation result for half-linear perturbation problems with mean curvature operator in Minkowski space. As applications of the abovementioned result, we shall prove the existence of nodal solutions for the following problem $\left\{\begin{array}{l}-\operatorname{div}\left(\nabla v / \sqrt{1-|\nabla v|^{2}}\right)=\alpha(|x|) v^{+}+\beta(|x|) v^{-}+\lambda a(|x|) f(v), \text { in } B_{R}(0), \text { where } \lambda \neq 0 \text { is a parameter, } R \text { is a } \\ v(x)=0, \text { on } z B_{R}(0),\end{array}\right.$ positive constant, and $B_{R}(0)=\left\{x \in \mathbb{R}^{N}:|x|<R\right\}$ is the standard open ball in the Euclidean space $\mathbb{R}^{N}(N \geq 1)$ which is centered at the origin and has radius $R . a(|x|) \in C[0, R]$ is positive, $v^{+}=\max \{v, 0\}, v^{-}=-\min \{v, 0\}, \alpha(|x|), \beta(|x|) \in C[0, R] ; f \in C(\mathbb{R}, \mathbb{R}), s f(s)$ $>0$ for $s \neq 0$, and $f_{0} \in[0, \infty]$, where $f_{0}=\lim _{|s| \longrightarrow 0} f(s) / s$. We use unilateral global bifurcation techniques and the approximation of connected components to prove our main results.

\section{Introduction}

We first consider the following problem with mean curvature operator in Minkowski space:

$$
\begin{cases}-\operatorname{div}\left(\frac{\nabla v}{\sqrt{1-|\nabla v|^{2}}}\right)=\lambda a(|x|) v+F(|x|, v, \lambda), & \text { in } B_{R}(0), \\ v(x)=0, & \text { on } \partial B_{R}(0),\end{cases}
$$

where $\lambda \neq 0$ is a parameter, $R$ is a positive constant, and $B_{R}(0)=\left\{x \in \mathbb{R}^{N}:|x|<R\right\}$ is the standard open ball in the Euclidean space $\mathbb{R}^{N}(N \geq 1)$, which is centered at the origin and has radius $R$. Here, the nonlinear function $F \in C\left(\overline{B_{R}(0)} \times \mathbb{R}^{2}, \mathbb{R}\right)$ and $a(|x|)$ is a weighted function. Dirichlet problem (1) is associated to the mean curvature operator in the flat Minkowski space $\mathbb{L}^{N+1}$ with $\left(x_{1}, \ldots, x_{N}, t\right)$ and the Lorentzian metric $\sum_{i=1}^{N}\left(d x_{i}\right)^{2}-(d t)^{2}$.

Some important and interesting results [1-3] for this type of problems have been obtained. Some specialists have studied problem (1); for example, Cheng and Yau [4] and Treibergs [5] studied problem (1) with $\lambda a v \equiv F \equiv 0$ and $\lambda a v+F \equiv C$, respectively. Bidaut-Véron and Ratto [6] and López [7] studied problem (1) with $a \equiv 0, F=f(u)$ and $a \equiv 0$, $F=k v+\lambda$, respectively.

Recently, Bereanu et al. $[8,9]$ have proved existence of classical positive radial solutions for problem (1) by Leray-Schauder degree argument and critical point theory.

In 2016, Ma et al. [10] and Dai and Wang $[11,12]$ studied the existence of radial positive solutions and radial nodal solutions for problem (1) (where $\lambda a v+g=\lambda f(|x|, v)$ ) by bifurcation techniques, respectively.

On the contrary, among the abovementioned papers, the nonlinearities are differentiable at the origin. In [13], Berestycki established an important global bifurcation theorem from intervals for a class of second-order problems involving nondifferentiable nonlinearity.

Recently, Dai and Ma $[14,15]$ considered interval bifurcation problem for second-order and high-dimensional $p$-Laplacian problems involving nondifferentiable nonlinearity, respectively. 
In 2016, when $a(x) \equiv 1, R \equiv 1$, Dai and Yang [16] have established a global bifurcation result from interval for the following problem with nondifferentiable nonlinearity:

$$
\begin{cases}-\Delta u=\lambda a(x) u+F(x, u, \lambda), & \text { in } B_{R}(0), \\ u(x)=0, & \text { on } \partial B_{R}(0) .\end{cases}
$$

It is clear that the radial solutions of (2) is equivalent to the solutions of the following problem:

$$
\left\{\begin{array}{l}
-\left(r^{N-1} u^{\prime}\right)^{\prime}=\lambda r^{N-1} a(r) u+r^{N-1} F(r, u, \lambda), \quad r \in(0, R), \\
u^{\prime}(0)=u(R)=0
\end{array}\right.
$$

where $\lambda$ is a parameter, $r=|x|$ and $x \in B_{R}(0)$, the nonlinear term $F$ has the form $F=f+g$, where $f, g \in C\left([0, R] \times \mathbb{R}^{2}\right)$ are radially symmetric with respect to $r$, and $a, f$, and $g$ satisfy the following conditions:

(H1) $a \in C([0, R],(0, \infty))$ is radially symmetric $(H 2)|(f(r, s, \lambda)) / s| \leq M_{1}$, for all $r \in(0, R), 0<|s| \leq R$ and all $\lambda \in \mathbb{R}$, where $M_{1}$ is a positive constant

(H3) $g(r, s, \lambda)=o(|s|)$ near $s=0$ uniformly in $r \in(0, R)$ and $\lambda$ on bounded sets

Let $C[0, R]$ is a real Banach space with the norm $\|u\|_{\infty}=\max _{r \in[0, R]}|u(r)|$. Let $E=\left\{u(r) \in C^{1}[0, \quad R]: u^{\prime}(0)=\right.$ $u(R)=0\}$ with the usual norm $\|u\|=\max \left\{\|u\|_{\infty},\left\|u^{\prime}\right\|_{\infty}\right\}$. Let $S_{k}^{+}$denote the set of functions in $E$ which have exactly $k-1$ interior nodal (i.e., nondegenerate) zeros in $(0, R)$ and are positive at $r=0$, and set $S_{k}^{-}=-S_{k}^{+}$and $S_{k}=S_{k}^{+} \cup S_{k}^{-}$. Let $\Phi_{k}^{ \pm}=$ $\mathbb{R} \times S_{k}^{ \pm}$under the product topology. We use $\mathcal{S}$ to denote the closure of the nontrivial solutions set of problem (3) in $\mathbb{R} \times E$, and $\mathcal{S}_{k}^{ \pm}$to denote the subset of $\mathcal{S}$ with $u \in \mathcal{S}_{k}^{ \pm}$and $\mathcal{S}_{k}=\mathcal{S}_{k}^{+} \cup \mathcal{S}_{k}^{-}$.

Using the same method to prove ([16, Theorem 1]) with obvious changes, we may get the following global bifurcation result about Lemma 1 .

Lemma 1 (see [16, Theorem 1]). Let (H1), (H2), and (H3) hold. Let $I_{k}=\left[\lambda_{k}-M_{1}, \lambda_{k}+M_{1}\right]$. The component $\mathscr{C}_{k}^{+}$of $\mathcal{S}_{k}^{+} \cup\left(I_{k} \times\{0\}\right)$, containing $I_{k} \times\{0\}$, is unbounded and lies in $S_{k}^{+} \cup\left(I_{k} \times\{0\}\right)$ and the component $\mathscr{C}_{k}^{-}$of $\mathcal{S}_{k}^{-} \cup\left(I_{k} \times\{0\}\right)$, containing $I_{k} \times\{0\}$, is unbounded and lies in $S_{k}^{-} \cup\left(I_{k} \times\{0\}\right)$.

Let $\lambda_{k}$ be the $k$ th eigenvalue of the following eigenvalue problem:

$$
\left\{\begin{array}{l}
-\left(r^{N-1} u^{\prime}\right)^{\prime}=\lambda r^{N-1} a(r) u, \quad r \in(0, R) \\
u^{\prime}(0)=u(R)=0
\end{array}\right.
$$

It is well known that $\lambda_{k}$ is positive and simple, and the eigenfunction corresponding to it has exactly $k-1$ simple zeros in $(0, R)$ (see [17, Theorem 2.1]).

Furthermore, Dai and Yang [16] $(a(x) \equiv 1, \quad R \equiv 1)$ established spectrum of the following problem:

$$
\left\{\begin{array}{l}
-\left(r^{N-1} u^{\prime}\right)^{\prime}=\lambda a(r) r^{N-1} u+\alpha(r) r^{N-1} u^{+}+\beta(r) r^{N-1} u^{-}, \quad r \in(0, R), \\
u^{\prime}(0)=u(R)=0
\end{array}\right.
$$

where $a$ satisfies $(H 1), u^{+}=\max \{u, 0\}, u^{-}=-\min \{u, 0\}, \alpha(r)$ and $\beta(r)$ satisfy:

(H4) $\alpha(r), \beta(r) \in C[0, R]$ are radially symmetric

Problem (5) is called half linear because it is positively homogeneous and linear in the cones $u>0$ and $u<0$. Similar to [13], we say that $\lambda$ is a half-eigenvalue of problem (5), if there exists a nontrivial solution $\left(\lambda, u_{\lambda}\right)$. The eigenvalue $\lambda$ is said to be simple if $v=c u_{\lambda}, c>0$, for all solutions $(\lambda, v)$ of problem (5).

Using the same method to prove ([16], Theorem 2) with obvious changes, we may get the following result about Lemma 2.
Lemma 2 (see [16, Theorem 2]). Let (H1) and (H4) hold. There exist two sequences of simple halfeigenvalues for problem (5), $\lambda_{1}^{+}<\lambda_{2}^{+} \ldots<\lambda_{k}^{+}<\ldots$ and $\lambda_{1}^{-}<\lambda_{2}^{-} \ldots<\lambda_{k}^{-}<\ldots$. The corresponding half-linear solutions are in $\left\{\lambda_{k}^{+}\right\} \times S_{k}^{+}$and $\left\{\lambda_{k}^{-}\right\} \times S_{k}^{-}$. Furthermore, aside from these solutions and the trivial one, there are no other solutions of problem (5).

Motivated by the abovementioned papers, in this paper, we shall firstly establish a Dancer-type unilateral global bifurcation result (see Theorem 2) for the following problem:

$$
\begin{cases}-\operatorname{div}\left(\frac{\nabla v}{\sqrt{1-|\nabla v|^{2}}}\right)=\lambda a(|x|) v+\alpha(|x|) v^{+}+\beta(|x|) v^{-}+g(|x|, v, \lambda), & \text { in } B_{R}(0), \\ v(x)=0, & \text { on } \partial B_{R}(0) .\end{cases}
$$


It is clear that the radial solutions of (6) is equivalent to the solutions of the following problem:

$$
\left\{\begin{array}{l}
-\left(r^{N-1} \frac{u^{\prime}}{\sqrt{1-u^{\prime 2}}}\right)^{\prime}=r^{N-1}\left(\lambda a(r) u+\alpha u^{+}\right. \\
\left.\quad+\beta u^{-}+g(r, u, \lambda)\right), \quad r \in(0, R), \\
u^{\prime}(0)=u(R)=0
\end{array}\right.
$$

where $\lambda \neq 0$ is a parameter, $r=|x|$ and $u(r)=v(|x|)$, $a$ satisfies $(H 1), \alpha, \beta$ satisfy $(H 4)$. Assume $g \in C([0, R] \times[-R, R] \times \mathbb{R})$ satisfies $(H 3)$ and the following condition:

(H5) There exists a function $h(t, u) \in C([0, R] \times$ $[-R, R], \mathbb{R})$ with $h(t, s) s>0$ for any $r \in[0, R]$ and $s \neq 0$, such that $g(t, u, \lambda)=\lambda h(t, u)$
By a solution to problem (7), we mean a function $u=u(r) \in C^{1}[0, R]$ with $\left\|u^{\prime}\right\|_{\infty}<1$, such that $r^{N-1} u^{\prime} / \sqrt{1-u^{\prime 2}}$ is differentiable and (7) is satisfied. Here, $\|\cdot\|_{\infty}$ denotes the usual sup-norm.

Furthermore, we shall investigate the existence of solutions for the following problems:

$$
\left\{\begin{array}{l}
-\operatorname{div}\left(\frac{\nabla v}{\sqrt{1-|\nabla v|^{2}}}\right)=\alpha(|x|) v^{+}+\beta(|x|) v^{-} \\
+\lambda a(|x|) f(v), \quad \text { in } B_{R}(0), \\
v(x)=0, \quad \text { on } \partial B_{R}(0) .
\end{array}\right.
$$

It is clear that the radial nodal solutions of (8) is equivalent to the solutions of the following problem:

$$
\left\{\begin{array}{l}
-\left(r^{N-1} \frac{u^{\prime}}{\sqrt{1-u^{\prime 2}}}\right)^{\prime}=\alpha r^{N-1} u^{+}+\beta r^{N-1} u^{-}+\lambda r^{N-1} a(r) f(u), \quad r \in(0, R), \\
u^{\prime}(0)=u(R)=0
\end{array}\right.
$$

where $\lambda \neq 0$ is a parameter, $a$ satisfies (H1) and $\alpha$ and $\beta$ satisfy $(H 4)$. Clearly, the nonlinear term of (9) is not necessarily differentiable at the origin because of the influence of the term $\alpha u^{+}+\beta u^{-}$. So, the bifurcation theory of $[11,12]$ cannot be applied directly to obtain our results. Fortunately, using the global interval bifurcation, we can obtain some results of the existence of radial solutions which extend the corresponding results of [12].

The rest of this paper is arranged as follows. In Section 2, we establish a unilateral global bifurcation result for halflinear perturbation problems (7). In Section 3, on the basis of the unilateral global bifurcation result (see Theorem 2), we shall investigate the existence of nodal solutions for a class of the half-linear perturbation problems (9).

\section{Unilateral Global Bifurcation for Problem (7)}

From Lemma 1 (or see Theorem 1 and its proof of [16]), we can easily get the following result.
Theorem 1. There exist two unbounded subcontinua $D_{k}^{+}$and $D_{k}^{-}$of solutions of (3) in $\mathbb{R} \times E$, bifurcating from $I_{k} \times\{0\}$, for $\nu \in\{+,-\}$, such that $D_{k}^{v} \subset\left(\Phi_{k}^{v} \cup\left(I_{k} \times\{0\}\right)\right)$.

Similar to the process of $([11,(2.1)-\mathrm{p} .60])$ or $([12,(2.1)-$ p.469]) obtained, problem (7) is equivalent to

$$
\left\{\begin{array}{l}
-\left(r^{N-1} \frac{u^{\prime}}{\sqrt{1-u^{\prime 2}}}\right)^{\prime}=r^{N-1} H_{1}(r, u, \lambda), \quad r \in(0, R), \\
u^{\prime}(0)=u(R)=0,
\end{array}\right.
$$

where $H_{1}(r, u, \lambda)=\lambda a(r) u+\alpha u^{+}+\beta u^{-}+g(r, u, \lambda)$. If $u$ is a solution of problem (7), then for any $r \in(0, R)$, one has that

$$
\begin{aligned}
r^{N-1} H_{1}(r, u, \lambda) & =-\left(r^{N-1} u^{\prime}\right)^{\prime} \frac{1}{\sqrt{1-u^{\prime 2}}}-r^{N-1} u^{\prime 2} u^{\prime \prime} \frac{1}{\left(1-u^{\prime 2}\right) \sqrt{1-u^{\prime 2}}} \\
& =-(N-1) r^{N-2} u^{\prime} \frac{1}{\sqrt{1-u^{\prime 2}}}-r^{N-1} u^{\prime \prime} \frac{1}{\sqrt{1-u^{\prime 2}}} \cdot \frac{1}{1-u^{\prime 2}} \\
& -u^{\prime \prime}
\end{aligned}
$$

By (11), one obtains that 
Substituting (12) into (11), it follows that

Thus, problem (7) is equivalent to

$-\left(r^{N-1} u^{\prime}\right)^{\prime}=r^{N-1} H_{1}(r, u, \lambda)\left(1-u^{\prime 2}\right)^{3 / 2}+(N-1) r^{N-2} u^{\prime 3}$.

$$
\left\{\begin{array}{l}
-\left(r^{N-1} u^{\prime}\right)^{\prime}=\lambda r^{N-1} a(r) u+r^{N-1}\left(\alpha u^{+}+\beta u^{-}\right)+r^{N-1} K_{1}(r, u, \lambda), \quad r \in(0, R) \\
u^{\prime}(0)=u(R)=0
\end{array}\right.
$$

where

$$
\begin{aligned}
K_{1}(r, u, \lambda)= & \left(\lambda a(r) u+\alpha u^{+}+\beta u^{-}\right)\left(\left(1-\left(u^{\prime}\right)^{2}\right)^{3 / 2}-1\right) \\
& +g(r, u, \lambda)\left(1-\left(u^{\prime}\right)^{2}\right)^{3 / 2}-\frac{N-1}{r} u^{\prime 3}
\end{aligned}
$$

In Theorem 2, we shall prove that $K_{1}(r, u, \lambda)$ satisfies (H3). Similar to ([13, (3.2)-p.383]), problem (14) is the perturbation of problem (5). In other words, problem (7) is the perturbation of problem (5).

Next, it is clear that problem (14) can be equivalently written as

$$
u=\lambda L_{N} u+L_{N} \circ K_{2}=F(\lambda, u),
$$

where

$$
\begin{aligned}
K_{2}(r, u, \lambda) & =\alpha u^{+}+\beta u^{-}+K_{1}(r, u, \lambda), \\
L_{N} u & =\int_{0}^{R} G(t, s) s^{N-1} a(s) u(s) \mathrm{d} s, \\
L_{N} \circ K_{2} & =\int_{0}^{R} G(t, s) s^{N-1} K_{2}(s, u(s), \lambda) \mathrm{d} s,
\end{aligned}
$$

where $G(r, s)$ be Green's function associated with the operator $L u$ : $=-\left(r^{N-1} u^{\prime}\right)^{\prime}$ with the same boundary condition as in problem (14) (see [18]). $L: E \longrightarrow E$ is linear completely continuous (see ([18, (2.5)-p.502 to line 4-p.503])). $L \circ K_{2}: \mathbb{R} \times E \longrightarrow E$ is completely continuous (see [18]). Moreover, $F$ is completely continuous from $\mathbb{R} \times E \longrightarrow E$ and $F(\lambda, 0)=0, \forall \lambda \in \mathbb{R}$.

Let

$$
\bar{g}(r, u, \lambda)=\max _{0 \leq|s| \leq u}|g(r, s, \lambda)|, \quad \text { for } r \in(0, R) \text { and } \lambda
$$$$
\text { on bounded sets, }
$$

then $\bar{g}$ is nondecreasing and

$$
\lim _{u \longrightarrow 0^{+}} \frac{\bar{g}(r, u, \lambda)}{u}=0,
$$

uniformly for $r \in(0, R)$ and $\lambda$ on bounded sets.

Similar to Lemma 2.2 in [12], with obvious changes, we may get the following lemma.
Lemma 3. For fixed $\lambda>0$, if $\left\{u_{k}\right\}$ is a sequence of solutions of problem (7) satisfying $\lim _{k} \longrightarrow+\infty u_{k}=0$ uniformly in $r \in[0, R]$, then $\lim _{k \longrightarrow+\infty} u_{k}^{\prime}=0$ and $\lim _{k \longrightarrow+\infty} u_{k}^{\prime \prime}=0$ uniformly in $r \in$ $[0, R]$.

Proof. Integrating the first equation of problem (7) from 0 to $r$ for any $r \in[0, R]$, we get that

$$
r^{N-1} \frac{u_{k}^{\prime}}{\sqrt{1-u_{k}^{\prime 2}}}=-\int_{0}^{r}\left[\alpha r^{N-1} u_{k}^{+}+\beta r^{N-1} u_{k}^{-}+r^{N-1} g\left(r, u_{k}, \lambda\right)\right] \mathrm{d} r
$$

By (H3), we have that $\lim _{k \longrightarrow+\infty} g\left(r, u_{k}, \lambda\right)=0$ uniformly $r \in[0, R]$ and $\lambda$ on bounded sets. It follows that $\lim _{k \longrightarrow+\infty} \mathcal{u}_{k}^{\prime \prime}=0$ uniformly in $r \in[0, R]$.

By (12), for any $r \in(0, R)$, one has that

$$
\begin{aligned}
-u_{k}^{\prime \prime}= & {\left[\lambda a(r) u_{k}+\alpha u_{k}^{+}+\beta u_{k}^{-}+g\left(r, u_{k}, \lambda\right)\right]\left(1-u_{k}^{\prime 2}\right)^{3 / 2} } \\
& +\frac{N-1}{r} u_{k}^{\prime}\left(1-u_{k}^{\prime 2}\right) .
\end{aligned}
$$

By $\lim _{k \longrightarrow+\infty} u_{k}=0$ and $\lim _{k \longrightarrow+\infty} u_{k}^{\prime}=0$ uniformly in $r \epsilon$ $[0, R]$ together with $(21)$, it follows that $\lim _{k \longrightarrow+\infty} u_{k}^{\prime}=0$ uniformly in $r \in(0, R)$.

Taking the limit $r \longrightarrow 0^{+}$on both sides of equation (21), together with $\lim _{r \longrightarrow 0^{+}} u_{k}(r)=0$ and $\lim _{r \longrightarrow 0^{+}} u_{k}^{\prime}(r)=0$, by L'Hospital's rule, we have that

$$
\begin{aligned}
& -\lim _{r \longrightarrow 0^{+}} u_{k}^{\prime \prime}(r)=\lim _{r \longrightarrow 0^{+}} \frac{(N-1) u_{k}^{\prime}(r)\left(1-u_{k}^{\prime 2}(r)\right)}{r} \\
& =\lim _{r \longrightarrow 0^{+}} \frac{\left.(N-1) u_{k}^{\prime \prime}(r)\left(1-u_{k}^{\prime 2}\right)(r)\right)+(N-1) u_{k}^{\prime}\left(-2 u_{k}^{\prime} u_{k}^{\prime \prime}\right)}{1} \\
& =\lim _{r \longrightarrow 0^{+}}(N-1) u_{k}^{\prime \prime}(r) .
\end{aligned}
$$

We obtain that $\lim _{r \rightarrow 0^{+}} u_{k}^{\prime \prime}(r)=0$.

By $\lim _{r \longrightarrow R^{-}} u_{k}(r)=\lim _{r \longrightarrow R^{-}} u_{k}^{\prime}(r)=0$, taking the limit $r \longrightarrow R^{-}$on both sides of equation (21), we may get that $\lim _{r \rightarrow R^{-}} u_{k}^{\prime \prime}(r)=0$.

Moreover, it follows that $\lim _{k \longrightarrow+\infty} u_{k}^{\prime \prime}=0$ uniformly in $r \in[0, R]$.

Lemma 4. For fixed $\lambda>0$, if $u$ is a solutions of problem (7) satisfying $u \longrightarrow 0$ uniformly in $r \in[0, R]$, then 


$$
\begin{aligned}
\lim _{u \longrightarrow 0^{+}} \frac{\left(1-\left(u^{\prime}\right)^{2}\right)^{3 / 2}-1}{u} & =0, \\
\lim _{u \longrightarrow 0^{+}} \frac{(N-1)\left(u^{\prime}\right)^{3}}{u} & =0 .
\end{aligned}
$$

Proof. From Lemma 3, we have that $\lim _{u \longrightarrow 0} u^{\prime}=0$ and $\lim _{u \longrightarrow 0} u^{\prime \prime}=0$ uniformly in $r \in[0, R]$.

By L'Hospital's rule, we have

$$
\begin{aligned}
\lim _{u \longrightarrow 0^{+}} \frac{\left(1-\left(u^{\prime}\right)^{2}\right)^{3 / 2}-1}{u} & =\lim _{u \longrightarrow 0^{+}} \frac{(3 / 2)\left(1-\left(u^{\prime}\right)^{2}\right)^{1 / 2} \cdot\left(-2 u^{\prime} u^{\prime \prime}\right)}{u^{\prime}}=\lim _{u \longrightarrow 0^{+}} \frac{3}{2}\left(1-\left(u^{\prime}\right)^{2}\right)^{1 / 2} \cdot\left(-2 u^{\prime \prime}\right)=0, \\
\lim _{u \longrightarrow 0^{+}} \frac{(N-1)\left(u^{\prime}\right)^{3}}{u} & =\lim _{u \longrightarrow 0^{+}} \frac{(N-1) 3\left(u^{\prime}\right)^{2} u^{\prime \prime}}{u^{\prime}}=\lim _{u \longrightarrow 0^{+}} 3(N-1) u^{\prime} u^{\prime \prime}=0 .
\end{aligned}
$$

Using a similar method to prove ([14, Theorem 3.3]) or ([15, Theorem 3.1]) with obvious changes, we may obtain the following result.

Theorem 2. Let (H1) and (H3)-(H6) hold. For $v \in\{+,-\}$, $\left(\lambda_{k}^{v}, 0\right)$ is a bifurcation point for problem (7). Moreover, there exists a subcontinuum $\mathscr{D}_{k}^{v}$ of solutions of problem (7), for $v \in\{+,-\}$, such that

(i) $\mathscr{D}_{k}^{v} \subset\left(\Phi_{k}^{v} \cup\left\{\left(\lambda_{k}^{v}, 0\right)\right\}\right)$;

(ii) $\mathscr{D}_{k}^{v}$ is unbounded;

(iii) $\left(\lambda_{k}^{v}, 0\right)$ is the unique bifurcation point for problem (7);

(iv) $\lim _{\lambda \longrightarrow \infty}\|u\|=\max \{1, R\}$ for $(\lambda, u) \in \mathscr{D}_{k}^{v} \backslash\left\{\left(\lambda_{k}^{v}, 0\right)\right\}$;

(v) $\lim _{k \longrightarrow \infty}\|u\|=0$ for $(\lambda, u) \in \mathscr{D}_{k}^{\nu} \backslash\left\{\left(\lambda_{k}^{v}, 0\right)\right\}$.

Proof. By (19), one obtains that

$\frac{|g(r, u, \lambda)|}{|u|} \leq \frac{\bar{g}(r, u, \lambda)}{|u|} \leq \frac{\bar{g}(r,|u|, \lambda)}{|u|} \longrightarrow 0, \quad$ as $|u| \longrightarrow 0$,

uniformly for $r \in(0, R)$ and $\lambda$ on bounded sets. It follows that

$$
\lim _{|u| \longrightarrow 0} \frac{g(r, u, \lambda)\left(1-\left(u^{\prime}\right)^{2}\right)^{3 / 2}}{|u|}=0 .
$$

By Lemma 4, we can get that

$$
\begin{array}{r}
\frac{\left(\lambda a(r) u+\alpha u^{+}+\beta u^{-}\right)\left(\left(1-\left(u^{\prime}\right)^{2}\right)^{3 / 2}-1\right)}{|u|} \longrightarrow 0, \\
\frac{(N-1)\left(u^{\prime}\right)^{3}}{r|u|} \longrightarrow 0, \quad \text { as }|u| \longrightarrow 0 .
\end{array}
$$

By (26) and (27), we have that

$$
\frac{K_{1}(r, u, \lambda)}{|u|} \longrightarrow 0, \quad \text { as }|u| \longrightarrow 0,
$$

uniformly for $r \in(0, R)$ and $\lambda$ on bounded sets. Let $\alpha^{0}:=\max _{r \in[0, R]}|\alpha(r)|$ and $\beta^{0}:=\max _{r \in[0, R]}|\beta(r)|$. For $0<|u| \leq R$, one get that $\left|\left(\alpha u^{+}+\beta u^{-}\right) / u\right| \leq \alpha^{0}+\beta^{0}$. Furthermore, let

$$
I_{k}^{0}=\left[\lambda_{k}-\frac{\alpha^{0}+\beta^{0}}{a_{0}}, \lambda_{k}+\frac{\alpha^{0}+\beta^{0}}{a_{0}}\right] \text {. }
$$

Theorem 1 shows that there exist two unbounded subcontinua $\mathscr{D}_{k}^{+}$and $\mathscr{D}_{k}^{-}$of solutions of (14) in $\mathbb{R} \times E$, bifurcating from $I_{k}^{0} \times\{0\}$, and $\mathscr{D}_{k}^{v} \subset\left(\Phi_{k}^{v} \cup\left(I_{k}^{0} \times\{0\}\right)\right)$ for $v=+$ and $v=-$, in other words, (i) and (ii) hold. steps:

We divide the rest of proofs into the following several

(iii) Let us show that $\mathscr{D}_{k}^{v} \cap(\mathbb{R} \times\{0\})=\left(\lambda_{k}^{v}, 0\right)$, i.e., $\left(\lambda_{k}^{v}, 0\right)$ is the unique bifurcation point for problem (7). Indeed, if there exists $\left(\lambda_{n}, u_{n}\right)$ be a sequence of solutions of problem (7) converging to $(\lambda, 0)$. By $\left\|u_{n}\right\|$ for the two side of (14), let $w_{n}=u_{n} /\left\|u_{n}\right\|$, then $w_{n}$ should be a solution of problem:

$$
\begin{aligned}
w_{n}= & L_{N}\left[\lambda r^{N-1} a(r) w_{n}+\alpha(r) r^{N-1} w_{n}^{+}+\beta(r) r^{N-1} w_{n}^{-}\right. \\
& \left.+\frac{r^{N-1} K_{1}\left(r, u_{n}, \lambda_{n}\right)}{\left\|u_{n}\right\|}\right]
\end{aligned}
$$

By (19), it follows that

$$
\begin{aligned}
\frac{\left|g\left(r, u_{n}, \lambda_{n}\right)\right|}{\left\|u_{n}\right\|} & \leq \frac{\bar{g}\left(r, u_{n}, \lambda_{n}\right)}{\left\|u_{n}\right\|} \leq \frac{\bar{g}\left(r,\left\|u_{n}\right\|_{\infty}, \lambda_{n}\right)}{\left\|u_{n}\right\|} \\
& \leq \frac{\bar{g}\left(r,\left\|u_{n}\right\|, \lambda_{n}\right)}{\left\|u_{n}\right\|} \longrightarrow 0, \quad \text { as }\left\|u_{n}\right\| \longrightarrow 0
\end{aligned}
$$

uniformly for $r \in(0, R)$ and $\lambda$ on bounded sets.

By proof of Theorem 1.1 of [11] or proof of Theorem 1.1 of [12], we have that 


$$
\begin{aligned}
\frac{(N-1)\left(u_{n}^{\prime}\right)^{3}}{r\left\|u_{n}\right\|} & \longrightarrow 0, \\
\frac{\left(\lambda_{n} a(r) u_{n}+\alpha(r) u_{n}^{+}+\beta(r) u_{n}^{-}\right)\left(\left(1-\left(u_{n}^{\prime}\right)^{2}\right)^{3 / 2}-1\right)}{\left\|u_{n}\right\|} & \longrightarrow 0 \\
\text { as }\left\|u_{n}\right\| & \longrightarrow 0 .
\end{aligned}
$$

By (31) and (32), we have that

$$
\frac{K_{1}\left(r, u_{n}, \lambda_{n}\right)}{\left\|u_{n}\right\|} \longrightarrow 0 \quad \text { as }\left\|u_{n}\right\| \longrightarrow 0,
$$

uniformly for $r \in(0, R)$ and $\lambda_{n}$ on bounded sets.

By (33) and the compactness of $L$, we obtain that, for some convenient subsequence $w_{n} \longrightarrow w_{0}$ as $n \longrightarrow+\infty$. Now, $w_{0}$ verifies the following equation:

$$
-\left(r^{N-1} w_{0}^{\prime}\right)^{\prime}=\lambda a(r) r^{N-1} w_{0}+\alpha(r) r^{N-1} w_{0}^{+}+\beta(r) r^{N-1} w_{0}^{-},
$$

and $\left\|w_{0}\right\|=1$. This implies that $\lambda=\lambda_{k}^{v}$ for $v \in\{+,-\}$.

(iv) Now, we shall prove that $\lim _{\lambda \longrightarrow \infty}\|u\|=\max \{1, R\}$. The idea is similar to the proof of Theorem 1.1 of [12], but we give a rough sketch of the proof for readers' convenience. We only prove the case of $v=+$ since the case of $v=-$ is similar. For any $\left(\lambda_{n}, u_{n}\right) \in \mathscr{D}_{k}^{\nu} \backslash\left\{\left(\lambda_{k}^{v}, 0\right)\right\}$ with $\lambda_{n} \longrightarrow+\infty$ as $n \longrightarrow+\infty$. Let $t_{0}^{n}=0<t_{1}^{n}<t_{2}^{n}<\cdots<t_{k-1}^{n}<t_{k}^{n}=R$ denote the zeros of $u_{n}$ and $r_{0}^{n} \in\left[t_{j}^{n}, t_{j+1}^{n}\right](j \in\{0, \ldots, k-1\})$ such that $\left|u_{n}^{\prime}\left(r_{0}^{n}\right)\right|=\left\|u_{n}^{\prime}\right\|_{\infty}$. Let $r_{1}^{n}=\min \left\{r \in\left(t_{j}^{n}, t_{j+1}^{n}\right): u_{n}^{\prime}(r)=0\right\}$ (see [12, lines 1-4 in p.472]). Letting $\rho_{n} \in\left(r_{1}^{n}, r_{0}^{n}\right)$ (or $\left.\rho_{n} \in\left(r_{0}^{n}, r_{1}^{n}\right)\right)$, and $\rho_{*}=\liminf _{n \longrightarrow+\infty} \rho_{n}, r_{1}^{\infty}=\liminf _{n \longrightarrow+\infty}$ $r_{1}^{n}$, by [12, line 23: p.472-line 25: p.473], one may obtain that $u_{n}\left(r_{1}^{\infty}+\rho\right) \geq \sigma_{0}$ for $n$ large enough and constant $\sigma_{0}>0$, where $\rho \in\left(0, \rho_{*}-r_{1}^{\infty}\right)$. By (H5), letting $h_{1}=$ $\min _{\left[r_{1}^{\infty}+\rho / 4, r_{1}^{\infty}+\rho\right] \times\left[\sigma_{0}, R\right]} h\left(t, u_{n}\right)$. It follows that $g\left(r, u_{n}\right.$, $\left.\lambda_{n}\right) \geq \lambda_{n} h_{1}$. Now, integrating the first equation of problem (7) from $r_{1}^{\infty}+\rho / 4$ to $r$ for any $r \in\left[r_{1}^{\infty}+\rho / 2, r_{1}^{\infty}+\rho\right]$ and $n$ large enough, we get that

$$
\begin{aligned}
r^{N-1} \frac{u_{n}^{\prime}}{\sqrt{1-u_{n}^{\prime 2}}}= & -\int_{r_{1}^{\infty}+(\rho / 4)}^{r} t^{N-1}\left[\lambda_{n} a(r) u_{n}+\alpha u_{n}^{+}+\beta u_{n}^{-}\right. \\
& \left.+g\left(r, u_{n}, \lambda_{n}\right)\right] \mathrm{d} t .
\end{aligned}
$$

Set $\quad a_{0}=\min _{t \in[0, R]} a(t), \quad \alpha^{0}:=\max _{t \in[0, R]}|\alpha(t)| \quad$ and $\beta^{0}:=\max _{t \in[0, R]}|\beta(t)|$. By simple computation, we can show that

$$
\left|\lambda_{n} a(r) u_{n}+\alpha u_{n}^{+}+\beta u_{n}^{-}+g\left(r, u_{n}, \lambda_{n}\right)\right| \geq\left|\lambda_{n} \sigma_{0}\left(a_{0}+h_{1}\right)-R\left(\alpha^{0}-\beta^{0}\right)\right| \text { for all } r \in[0, R]
$$

Moreover, we have that

$$
\begin{aligned}
\frac{1}{\sqrt{1-\left\|u_{n}^{\prime}\right\|_{\infty}^{2}}} & \geq \frac{1}{\sqrt{1-u_{n}^{\prime 2}}} \geq\left|\frac{u_{n}^{\prime}}{\sqrt{1-u_{n}^{\prime 2}}}\right| \\
& =\left|\frac{1}{r^{N-1}} \int_{r_{1}^{\infty}+\rho / 4}^{r} r^{N-1}\left[\lambda_{n} a(r) u+\alpha u_{n}^{+}+\beta u_{n}^{-}+g\left(r, u_{n}, \lambda_{n}\right)\right] \mathrm{d} r\right| \\
& \geq \frac{\left|\lambda_{n} \sigma_{0}\left(a_{0}+h_{1}\right)-R\left(\alpha^{0}-\beta^{0}\right)\right|}{N r^{N-1}} \int_{r_{1}^{\infty}+\rho / 4}^{r} t^{N-1} \mathrm{~d} t \\
& =\frac{\left|\lambda_{n} \sigma_{0}\left(a_{0}+h_{1}\right)-R\left(\alpha^{0}-\beta^{0}\right)\right|}{r^{N-1}}\left(r^{N}-\left(r_{1}^{\infty}+\rho / 4\right)^{N}\right) \\
& \geq \frac{\left|\lambda_{n} \sigma_{0}\left(a_{0}+h_{1}\right)-R\left(\alpha^{0}-\beta^{0}\right)\right|}{N\left(r_{1}^{\infty}+\rho\right)^{N-1}}\left(\left(r_{1}^{\infty}+\rho / 2\right)^{N}-\left(r_{1}^{\infty}+\rho / 4\right)^{N}\right) .
\end{aligned}
$$

By $\quad \lim _{n \longrightarrow+\infty} \lambda_{n}=+\infty, \quad$ it follows that $\quad$ (v) Finally, we show that $\lim _{n \longrightarrow+\infty}\left\|u_{n}^{\prime}\right\|_{\infty}=1$. Noting that

$$
\left|u_{n}(r)\right|=\left|\int_{R}^{r} u_{n}(t) \mathrm{d} t\right| \leq \int_{R}^{r}\left|u_{n}(t)^{\prime}\right| \mathrm{d} t \leq\left\|u_{n}^{\prime}\right\|_{\infty} R .
$$

Thus, one may obtain that $\lim _{\lambda \longrightarrow \infty}\|u\|=\max \{1, R\}$.

$$
\lim _{k \longrightarrow \infty}\left\|u_{k}\right\|=0 .
$$

By Lemma 4, we only show that $\lim _{k \rightarrow \infty}\left\|u_{k}\right\|_{\infty}=0$. On the contrary, one supposes $\lim _{k \rightarrow \infty}\left\|u_{k}\right\|_{\infty}=\rho>0$. 
Let $t_{k}^{0}=0<t_{k}^{1}<t_{k}^{2}<\cdots<t_{k}^{k-1}<t_{k}^{k}=R$ denote the zeros of $u_{k}$. By [12, p. 475], there exists $r_{k} \in\left[t_{k}^{j}, t_{k}^{j+1}\right](j \in\{0, \ldots$, $k-1\})$ such that $\left|u_{k}\left(r_{k}\right)\right|=\left\|u_{k}\right\|_{\infty}=\rho_{k}$, where $\rho_{k} \geq \rho / 2$ for any $k \geq k_{0}, k_{0} \in \mathbb{N}$. Let $r_{0}^{k} \in\left(r_{k}, t_{k}^{j+1}\right)$ satisfy $u_{k}\left(r_{0}^{k}\right)=\theta \rho_{k}$, where $\theta \in(0,1)$. Set $a^{0}=\max _{t \in[0, R]} a(t)$, where $h^{1}:=\max _{(t, u) \epsilon}$ $[0, R] \times[-R, R]\left|h\left(t, u_{k}\right)\right|$. By $[12$, p. 475$]$, one may obtain that

$$
\begin{aligned}
u_{k}^{\prime} & \geq \frac{u_{k}^{\prime}}{\sqrt{1-u_{k}^{\prime 2}}}=\frac{1}{r^{N-1}} \int_{r_{k}}^{r} r^{N-1}\left[\lambda a(r) u_{k}+\alpha u_{k}^{+}+\beta u_{k}^{-}+g\left(r, u_{k}, \lambda\right)\right] \mathrm{d} r \\
& \geq-\frac{\lambda\left(R a^{0}+h^{1}\right)+R\left(\alpha^{0}+\beta^{0}\right)}{N r^{N-1}} \int_{r_{k}}^{r} t^{N-1} \mathrm{~d} t \\
& \geq-\frac{\lambda\left(R a^{0}+h^{1}\right)+R\left(\alpha^{0}+\beta^{0}\right)}{N} r .
\end{aligned}
$$

Furthermore, we have that

$$
\begin{aligned}
-(1-\theta) \rho_{k}= & u_{k}\left(r_{0}^{k}\right)-\rho_{k} \geq-\frac{\lambda\left(R a^{0}+h^{1}\right)+R\left(\alpha^{0}+\beta^{0}\right)}{N} \\
& \cdot \frac{1}{2}\left[\left(r_{0}^{k}\right)^{2}-r_{k}^{2}\right] .
\end{aligned}
$$

Therefore, we may obtain that

$$
t_{k}^{j+1}-t_{k}^{j}>r_{0}^{k}-r_{k} \geq \frac{N(1-\theta) \rho}{2\left[\lambda\left(R a^{0}+h^{1}\right)+R\left(\alpha^{0}+\beta^{0}\right)\right] R}>0 .
$$

However, by an argument similar to that of [19, Proposition 3.7], one obtains that $t_{k}^{j+1}-t_{k}^{j} \leq C / k \longrightarrow 0$, as $k \longrightarrow+0$, and we get a contradiction.

Thus, we have that $\limsup _{k \rightarrow+\infty}\left\|u_{k}\right\|_{\infty}=\rho=0$.

Remark 1. Theorem 2 indicates that the bifurcation interval $I_{k}^{0}=\left\{\lambda_{k}^{+}, \lambda_{k}^{-}\right\}$, i.e., for problem (7), the bifurcation interval $I_{k}^{0}$ is a finite point set. What conditions can ensure that the component indeed bifurcating from an interval is still an open problem for the problems with the mean curvature operator in Minkowski space.

To prove Theorem 4, we use Whyburn type superior limit theorems. From [20], if the collection of the infinite sequence of sets is unbounded, Whyburn's limit theorem ([21, Theorem 9.1]) cannot be used directly because the collection may not be relatively compact (where the definitions of superior limit and inferior limit, see [20, line 11 to line 16]). Dai [20] overcomed this difficulty and established the following results.

Lemma 5 (see [20, Lemma 2.5]). Let $X$ be a normal space and let $\left\{C_{n} \mid n=1,2, \ldots\right\}$ be a sequence of unbounded connected subsets of $X$. Assume that

(i) There exists $z^{*} \in \lim \inf _{n \longrightarrow+\infty} C_{n}$ with $\left\|z^{*}\right\|<+\infty$.

(ii) For every $R>0,\left(\cup_{n=1}^{+\infty} C_{n}\right) \cap \bar{B}_{R}$ is a relatively compact set of $X$, where

$$
B_{R}=\{x \in X \mid\|x\| \leq R\} .
$$

Then, $\mathbb{D}:=\limsup _{n \rightarrow \infty} C_{n}$ is unbounded, closed, and connected.

In order to treat the problems with nonasymptotic nonlinearity at $\infty$, we shall need the following lemmas.

Lemma 6 (see [20, Corollary 2.1]). Let X be a normal vector space and let $\left\{C_{n} \mid n=1,2, \ldots\right\}$ be a sequence of unbounded connected subsets of $X$. Assume that

(i) There exists $z^{*} \in \lim$ inf $_{n \longrightarrow+\infty} C_{n}$ with $\left\|z^{*}\right\|=+\infty$.

(ii) There exists a homeomorphism $T: X \longrightarrow X$ such that $\left\|T\left(z^{*}\right)\right\|<+\infty$ and $\left\{T\left(C_{n}\right)\right\}$ be a sequence of unbounded connected subsets in $X$.

(iii) For every $R>0,\left(\cup_{n=1}^{+\infty} C_{n}\right) \cap \bar{B}_{R}$ is a relatively compact set of $X$, where

$$
B_{R}=\{x \in X \mid\|x\| \leq R\} .
$$

Then, $\mathbb{D}:=\limsup _{n \rightarrow \infty} C_{n}$ is unbounded, closed, and connected.

Lemma 7 (see [20, Lemma 2.6]). Let (X, $\rho)$ be a metric space. If $\left\{C_{i}\right\}_{i \in \mathbb{N}}$ is a sequence of sets whose limit superior is $L$ and there exists a homeomorphism $T: X \longrightarrow X$ such that, for every $R>0,\left(\cup_{i=1}^{+\infty} T\left(C_{i}\right)\right) \cap \bar{B}_{R}$ is a relatively compact set, then for each $\epsilon>0$ there exists an $m$ such that, for every $n>m$, $C_{n} \subset V_{\epsilon}(L)$, where $V_{\epsilon}(L)$ denotes the set of all points $p$ with $\rho(p, x)<\epsilon$ for any $x \in L$.

\section{Nodal Solutions for Problem (9)}

Following Theorem 2, we shall investigate the existence of nodal solutions for problem (9), where $a(t), \alpha(t)$, and $\beta(t)$ satisfy conditions $(H 1)$ and (H4), respectively. We assume that $f$ satisfies the following assumptions:

(H6) $s f(s)>0$ for $s \neq 0$.

(H7) $f_{0} \in(0, \infty)$.

(H8) $f_{0}=\infty$.

(H9) $f_{0}=0$, where 


$$
f_{0}=\lim _{|s| \longrightarrow 0} \frac{f(s)}{s}
$$

Applying Theorem 2 to problem (1.10), we have the following results.

Theorem 3. Let (H1), (H4), (H6), and (H7) hold. For $v \in\{+,-\}$, $\left(\lambda_{k}^{v} / f_{0}, 0\right)$ is a bifurcation point for problem (9). Moreover, there exists an unbounded continuum $\mathscr{D}^{v}$ of solutions of problem (9), for $v \in\{+,-\}$ such that $\mathscr{D}_{k}^{v}$ joins $\left(\lambda_{k}^{v} / f_{0}, 0\right)$ to $(\infty$, $\max \{1, \quad R\}), \quad \mathscr{D}_{k}^{v} \subset\left(\left(\mathbb{R} \times S_{k}^{v}\right) \cup\left\{\lambda_{k}^{v} / f_{0}, 0\right\}\right), \quad$ and $\mathscr{D}_{k}^{v} \cap(\mathbb{R} \times\{0\})=\left(\lambda_{k}^{v} / f_{0}, 0\right)$. Furthermore, one obtains that $\lim _{k \longrightarrow \infty}\|u\|=0$ for $\left(\lambda, u_{\lambda}\right) \in \mathscr{D}_{k}^{\nu} \backslash\left\{\left(\left(\lambda_{k}^{v} / f_{0}\right), 0\right)\right\}$.

Theorem 4. Let (H1), (H4), (H6), and (H8) hold. For $v \in\{+$, $-\},(0,0)$ is a bifurcation point for problem (9). Moreover, there exists an unbounded continuum $\mathscr{D}_{k}^{\nu}$ of solutions of problem (9), for $v \in\{+,-\}$ such that $\mathscr{D}_{k}^{v}$ joins $(0,0)$ to $(\infty$, max $\{1, \quad R\}), \quad \mathscr{D}_{k}^{v} \subset\left(\left(\mathbb{R} \times S_{k}^{v}\right) \cup\{(0,0)\}\right)$, and $\mathscr{D}_{k}^{v} \cap(\mathbb{R} \times\{0\})=$ $\{(0,0)\}$. Furthermore, one obtains that $\lim _{k} \longrightarrow \infty\|u\|=0$ for $(\lambda, u) \in \mathscr{D}_{k}^{v} \backslash\{(0,0)\}$.

Theorem 5. Let (H1), (H4), (H6), and (H9) hold. ( $), 0)$ is a bifurcation point for problem (9). Moreover, there exists an unbounded continuum $\mathscr{D}_{k}^{\nu}$ of solutions of problem (9), for $v \in\{+,-\}$ and such that $\mathscr{D}_{k}^{v}$ joins $(\infty, 0)$ to $(\infty, \max \{1, R\})$, $\mathscr{D}_{k}^{v} \subset\left(\mathbb{R} \times S_{k}^{v}\right)$, and $\operatorname{Proj}_{\mathbb{R}}\left(\mathscr{D}_{k}^{v}\right) \neq \varnothing$. Furthermore, one obtain that $\lim _{k \longrightarrow \infty}\|u\|=0$ for $(\lambda, u) \in \mathscr{D}_{k}^{v}$.

From Theorems 3-5, we can easily derive the following corollary, which give the ranges of parameter guaranteeing problem (9) has zero, two, or four radial nodal solutions.

Corollary 1. Assume that (H1), (H2), (H6), and (H7) hold. We may get the following results:

(i) If $\lambda_{k}^{v}>0$ for $v \in\{+,-\}$, then there exists $\mu_{1} \in\left(0, \lambda_{k}^{v} / f_{0}\right)$ such that problem (9) has no radial solution for all $\lambda \in\left(0, \mu_{1}\right)$ and has at least two radial nodal solutions for all $\lambda \in\left(\mu_{1},+\infty\right)$

(ii) If $\nu \lambda_{k}^{v}>0$ for $\nu \in\{+,-\}$, then there exist $\mu_{1}^{+} \in$ $\left(0, \lambda_{k}^{+} / f_{0}\right)$ and $\mu_{1}^{-} \in\left(\lambda_{k}^{-} / f_{0}, 0\right)$ such that problem (9) has no radial solution for all $\lambda \in\left(\mu_{1}^{-}, 0\right) \cup\left(0, \mu_{1}^{+}\right)$and has at least two radial nodal solutions for all $\lambda \in\left(-\infty, \mu_{1}^{-}\right) \cup\left(\mu_{1}^{+},+\infty\right)$

(iii) If $v \lambda_{k}^{v}<0$ for $v \in\{+,-\}$, then there exist $\mu_{1}^{-} \in\left(0, \lambda_{k}^{+} / f_{0}\right)$ and $\mu_{1}^{+} \in\left(\lambda_{k}^{-} / f_{0}, 0\right)$ such that problem (9) has no radial solution for all $\lambda \in\left(\mu_{1}^{+}, 0\right) \cup\left(0, \mu_{1}^{-}\right)$and has at least two radial nodal solutions for all $\lambda \in\left(-\infty, \mu_{1}^{+}\right) \cup\left(\mu_{1}^{-},+\infty\right)$

(iv) If $\lambda_{k}^{v}<0$ for $v \in\{+,-\}$, then there exists $\mu_{1} \in\left(\lambda_{k}^{v} / f_{0}, 0\right)$ such that problem (9) has no radial solution for all $\lambda \in\left(\mu_{1}, 0\right)$ and has at least two radial nodal solutions for all $\lambda \in\left(-\infty, \mu_{1}\right)$

Corollary 2. Assume that (H1), (H2), (H6), and (H8) hold. We may get the following results:

(i) If $\lambda_{k}^{v}>0$ for $v \in\{+,-\}$, then problem (9) has at least two radial nodal solutions for all $\lambda \in(0,+\infty)$

(ii) If $\nu \lambda_{k}^{v} \neq 0$ for $\nu \in\{+,-\}$, then problem (9) has at least two radial nodal solutions for all $\lambda \in(-\infty, 0) \cup(0$, $+\infty)$

(iii) If $\lambda_{k}^{v}<0$ for $v \in\{+,-\}$, then problem (9) has at least two radial nodal solutions for all $\lambda \in(-\infty, 0)$

Corollary 3. Assume that (H1), (H2), (H6), and (H9) hold. We may get the following results:

(i) If $\lambda_{k}^{v}>0$ for $v \in\{+,-\}$, then there exists $0<\mu_{2} \leq \mu_{3}$ such that problem (9) has no radial solution for all $\lambda \in\left(0, \mu_{2}\right)$ and has at least four radial nodal solutions for all $\lambda \in\left(\mu_{3},+\infty\right)$

(ii) If $\nu \lambda^{\nu} \neq 0$ for $\nu \in\{+,-\}$, then there exists $0 \neq \nu \mu_{2}^{\nu} \leq \nu \mu_{3}^{v}$ such that problem (9) has no radial solution for all $\lambda \in\left(\mu_{2}^{-v}, 0\right) \cup\left(0, \mu_{2}^{v}\right)$ and has at least four radial nodal solutions for all $\lambda \in\left(-\infty, \mu_{3}^{-v}\right) \cup\left(\mu_{3}^{v},+\infty\right)$

(iii) If $\lambda_{k}^{v}<0$ for $v \in\{+,-\}$, then there exists $\mu_{3} \leq \mu_{2}<0$ such that problem (9) has no radial solution for all $\lambda \in\left(\mu_{2}, 0\right)$ and has at least four radial nodal solutions for all $\lambda \in\left(-\infty, \mu_{3}\right)$

Proof of Theorem 3. Let $\zeta \in C(\mathbb{R}, \mathbb{R})$ be such that

$$
f(u)=f_{0} u+\zeta(u),
$$

with

$$
\lim _{|u| \longrightarrow 0} \frac{\zeta(u)}{u}=0
$$

By [11, (2.1)-p. 60] or [12, (2.1)-p. 469], problem (9) is equivalent to

$$
\left\{\begin{array}{l}
-\left(r^{N-1} u^{\prime}\right)^{\prime}=\lambda r^{N-1} a(r) f_{0} u+r^{N-1}\left(\alpha u^{+}+\beta u^{-}\right)+r^{N-1} K_{3}(r, u, \lambda), \quad r \in(0, R) \\
u^{\prime}(0)=u(R)=0
\end{array}\right.
$$

where 


$$
K_{3}(r, u, \lambda)=\left(\lambda a(r) f_{0} u+\alpha u^{+}+\beta r^{N-1} u^{-}\right)\left(\left(1-\left(u^{\prime}\right)^{2}\right)^{3 / 2}-1\right)+\lambda a(r) \zeta(u)\left(1-\left(u^{\prime}\right)^{2}\right)^{3 / 2}-\frac{N-1}{r} u^{\prime 3}
$$

Using a similar method to prove (28) with obvious changes, we have that

$$
\frac{K_{3}(r, u, \lambda)}{|u|} \longrightarrow 0, \quad \text { as }|u| \longrightarrow 0,
$$

uniformly for $r \in(0, R)$ and $\lambda$ on bounded sets.

Let us consider problem (48) as a bifurcation problem from the trivial solution axis.

Applying Theorem 2 to problem (48), we have the following result.
For $v \in\{+,-\},\left(\lambda_{k}^{v} / f_{0}, 0\right)$ is a bifurcation point for problem (48). Moreover, there exists an unbounded continuum $\mathscr{D}_{k}^{v}$ of solutions of problem (48), such that $\mathscr{D}_{k}^{v}$ joins $\left(\lambda_{k}^{v} / f_{0}, 0\right)$ to $(\infty, \max \{1, R\}), D_{k}^{v} \subset\left(\left(\mathbb{R} \times S_{k}^{v}\right) \cup\left\{\left(\lambda_{k}^{v} / f_{0}, 0\right)\right\}\right)$, $D_{k}^{\nu} \cap(\mathbb{R} \times\{0\})=\left(\lambda_{k}^{v} / f_{0}, 0\right)$, and $\quad \lim _{k \longrightarrow \infty}\|u\|=0$ for $\left(\lambda, u_{\lambda}\right) \in \mathscr{D}_{k}^{\nu} \backslash\left\{\left(\lambda_{k}^{v} / f_{0}, 0\right)\right\}$.

Proof of Theorem 4. Inspired by the idea of [22], we define the cut-off function of $f$ as the following:

$$
f^{[n]}(s):= \begin{cases}n s, & s \in\left[-\frac{1}{n}, \frac{1}{n}\right], \\ {\left[f\left(\frac{2}{n}\right)-1\right](n s-2)+f\left(\frac{2}{n}\right),} & s \in\left(\frac{1}{n}, \frac{2}{n}\right), \\ -\left[f\left(-\frac{2}{n}\right)+1\right](n s+2)+f\left(-\frac{2}{n}\right), & s \in\left(-\frac{2}{n},-\frac{1}{n}\right), \\ f(s), & s \in\left(-\infty,-\frac{2}{n}\right] \cup\left[\frac{2}{n},+\infty\right) .\end{cases}
$$

We consider the following problem:

$$
\left\{\begin{array}{l}
-\left(r^{N-1} \frac{u^{\prime}}{\sqrt{1-u^{\prime 2}}}\right)^{\prime}=\alpha r^{N-1} u^{+}+\beta r^{N-1} u^{-}+\lambda r^{N-1} a(r) f^{[n]}(u), \quad r \in(0, R), \\
u^{\prime}(0)=u(R)=0 .
\end{array}\right.
$$

Clearly, we can see that $\lim _{n \longrightarrow+\infty} f^{[n]}(s)=f(s)$, $\left(f^{[n]}\right)_{0}=n$.

Similar to the proof of Theorem 3, there exists an unbounded continuum $\mathscr{D}_{k}^{v[n]}$ of solutions of problem (52) emanating from $\left(\lambda_{k}^{v} / n, 0\right)$ and joining to $(\infty, \max \{1, R\})$ such that $\mathscr{D}_{k}^{\nu[n]} \subset\left(\left(\mathbb{R} \times S_{k}^{v}\right) \cup\left\{\left(\lambda_{k}^{v} / n, 0\right)\right\}\right)$.

Taking $z^{*}=(0,0)$, we easily obtain that $z^{*} \in \liminf _{n \longrightarrow+\infty} \mathscr{D}_{k}^{v[n]}$. So, condition (i) in Lemma 5 is satisfied with $z^{*}=(0,0)$.

Since $F$ is completely continuous from $\mathbb{R} \times E \longrightarrow E$, we have that $\left(\cup_{n=1}^{\infty} \mathscr{D}_{k}^{\nu[n]}\right) \cap \bar{B}_{R}$ is precompact, and accordingly (ii) in Lemma 5 holds.

Therefore, by Lemma 5, $\mathscr{D}_{k}^{v}=\limsup _{n \rightarrow \infty} \mathscr{D}_{k}^{v[n]}$ is unbounded closed connected such that $z^{*} \in \mathscr{D}_{k}^{v}$, and $(\infty, \max \{1, R\}) \in \mathscr{D}_{k}^{v}$. Clearly, $u$ is the solution of problem (9) for any $(\lambda, u) \in \mathscr{D}_{k}^{v}$. From the definition of superior limit (see [21, P. 7]), we can easily see that $\mathscr{D}_{k}^{v} \subseteq \cup_{n=1}^{\infty} \mathscr{D}_{k}^{\nu[n]}$. So, one has that $\mathscr{D}_{k}^{v} \subset\left(\left(\mathbb{R} \times S_{k}^{v}\right) \cup\{(0,0)\}\right)$.

We may claim that $\mathscr{D}_{k}^{v} \cap(\mathbb{R} \times\{0\})=\{(0,0)\}$, i.e., $z^{*}=(0$, $0)$, is the unique bifurcation point of $\mathscr{D}_{k}^{v}$. Suppose on the contrary that there exists a sequence $\left(\lambda_{n}, u_{n}\right) \in \mathscr{D}_{k}^{v} \backslash\{(0,0)\}=$ $\limsup _{n \longrightarrow \infty} \mathscr{D}_{k}^{\nu[n]} \backslash\{(0,0)\}$ such that $\lim _{n \longrightarrow \infty} \lambda_{n} \neq 0$ and $\lim _{n \longrightarrow \infty} u_{n}=0$. Hence, for any $N_{0} \in \mathbb{N}$, there exists $n_{0} \geq N_{0}$ such that $\left(\lambda_{n}, u_{n}\right) \in \mathscr{D}_{k}^{v\left[n_{0}\right]}$. By (52), it follows that $\lambda_{n_{0}}=\lambda_{k}^{v} / n_{0}$ for $n_{0} \geq N_{0}$. From the arbitrary of $N_{0}$, it implies that $n_{0} \longrightarrow \infty$, i.e., $\mu=0$, which contradicts the assumption of $\mu \neq 0$.

By $\mathscr{D}_{k}^{\nu} \subseteq \cup_{n=1}^{\infty} \mathscr{D}_{k}^{\nu[n]}$ and Theorem 3, one obtains that $\lim _{k \longrightarrow \infty}\|u\|=0$ for $(\lambda, u) \in \mathscr{D}_{k}^{\nu} \backslash\{(0,0)\}$.

Proof of Theorem 5. Inspired by the idea of [22], we define the cut-off function of $f$ as the following: 


$$
f^{[n]}(s):= \begin{cases}\frac{1}{n} s, & s \in\left[-\frac{1}{n}, \frac{1}{n}\right], \\ {\left[f\left(\frac{2}{n}\right)-\frac{1}{n^{2}}\right](n s-2)+f\left(\frac{2}{n}\right),} & s \in\left(\frac{1}{n}, \frac{2}{n}\right), \\ -\left[f\left(-\frac{2}{n}\right)+\frac{1}{n^{2}}\right](n s+2)+f\left(-\frac{2}{n}\right), & s \in\left(-\frac{2}{n},-\frac{1}{n}\right), \\ f(s), & s \in\left(-\infty,-\frac{2}{n}\right] \cup\left[\frac{2}{n},+\infty\right) .\end{cases}
$$

We consider the following problem:

$$
\left\{\begin{array}{l}
-\left(r^{N-1} \frac{u^{\prime}}{\sqrt{1-u^{\prime 2}}}\right)^{\prime}=\alpha r^{N-1} u^{+}+\beta r^{N-1} u^{-}+\lambda r^{N-1} a(r) f^{[n]}(u), \quad r \in(0, R), \\
u \prime(0)=u(R)=0 .
\end{array}\right.
$$

Clearly, we can see that $\lim _{n \longrightarrow+\infty} f^{[n]}(s)=f(s)$, $\left(f^{[n]}\right)_{0}=1 / n$.

Similar to the proof of Theorem 3, there exists an unbounded continuum $\mathscr{D}_{k}^{v[n]}$ of solutions of the problem (54) emanating from $\left(\lambda_{k}^{\nu} n, 0\right)$, such that $\mathscr{D}_{k}^{\nu[n]} \subset\left(\left(\mathbb{R} \times S_{k}^{\nu}\right) \cup\right.$ $\left.\left\{\left(\lambda^{\nu} n, 0\right)\right\}\right)$ and $(\infty, \max \{1, R\}) \in \mathscr{D}_{k}^{\nu[n]}$.

Taking $z^{*}=(\infty, 0)$, we easily obtain that $z^{*} \in \liminf _{n \longrightarrow+\infty} \mathscr{D}^{\nu[n]}$ with $\left\|z^{*}\right\|_{\mathbb{R} \times E}=+\infty$ So, condition

(ii) in Lemma 6 is satisfied with $z^{*}=(+\infty, 0)$.

Define a mapping $T: R \times X \longrightarrow R \times X$ such that

$$
T(\lambda, u)= \begin{cases}\left(\frac{1}{\lambda}, u\right), & \lambda \in(-\infty, 0) \cup(0,+\infty), \\ (0, u), & \lambda=\infty, \\ (\infty, u), & \lambda=0 .\end{cases}
$$

It is easy to verify that $T$ is a homeomorphism and $\left\|T\left(z^{*}\right)\right\|_{\mathbb{R} \times X}=0$. Obviously, $\left\{T\left(\mathscr{D}_{k}^{v[n]}\right)\right\}$ be a sequence of unbounded connected subsets in $X$. So, (ii) in Lemma 6 holds. Since $F$ is completely continuous from $\mathbb{R} \times E \longrightarrow E$, we have that $\left(\cup_{n=1}^{+\infty} T\left(\mathscr{D}^{\nu[n]}\right)\right) \cap \bar{B}_{R}$ is precompact, and accordingly (iii) in Lemma 6 holds.

Therefore, by Lemma $6, \mathscr{D}_{k}^{v}=\limsup _{n \rightarrow \infty} \mathscr{D}_{k}^{v[n]}$ is unbounded closed connected such that $z^{*} \in \mathscr{D}_{k}^{\nu}$ and $(\infty, \max \{1, R\}) \in \mathscr{D}_{k}^{v}$. Obviously, $u$ is the solution of problem (9) for any $(\lambda, u) \in \mathscr{D}_{k}^{v}$. From the definition of superior limit (see [21, P. 7]), we can easily see that $\mathscr{D}_{k}^{\nu} \subseteq \cup_{n=1}^{\infty} \mathscr{D}_{k}^{\nu[n]}$. So, one has that $\mathscr{D}_{k}^{v} \subset\left(\mathbb{R} \times\left(S_{k}^{v} \cup\{0\}\right)\right)$. Next, we show that $\mathscr{D}_{k}^{v} \cap(\mathbb{R} \times\{0\})=\varnothing$. Suppose on the contrary that there exists a sequence $\left\{\left(\lambda_{n}, u_{n}\right)\right\} \subseteq \mathscr{D}_{k}^{v}$ such that $\lim _{n \longrightarrow+\infty} \lambda_{n}=\mu$ and $\lim _{n \longrightarrow+\infty}\left\|u_{n}\right\|=0$. From (9), we can easily get that

$$
\begin{aligned}
u_{n}= & \int_{0}^{R} G(r, s) s^{N-2} \\
& \cdot\left[\left(s \alpha u_{n}^{+}+s \beta u_{n}^{-}+\lambda_{n} a(r) s f\left(u_{n}\right)\right)\left(1-\left(u_{n}^{\prime}\right)^{2}\right)^{3 / 2}-(N-1) u_{n}^{\prime 3}\right] \mathrm{d} r .
\end{aligned}
$$

Letting $w_{n}=u_{n} /\left\|u_{n}\right\|$, we have that

$$
\begin{aligned}
w_{n}= & \int_{0}^{R} G(r, s) s^{N-2}\left[\left(s \alpha w_{n}^{+}+s \beta w_{n}^{-}\right.\right. \\
& \left.\left.+\lambda_{n} a(r) s \frac{f\left(u_{n}\right)}{\left\|u_{n}\right\|}\right)\left(1-\left(u_{n}^{\prime}\right)^{2}\right)^{3 / 2}-(N-1) \frac{u_{n}^{\prime 3}}{\left\|u_{n}\right\|}\right] \mathrm{d} r .
\end{aligned}
$$

Similar to (31), we can show that

$$
\lim _{n \longrightarrow+\infty} \frac{f\left(u_{n}\right)}{\left\|u_{n}\right\|}=0 .
$$

By (32), (57), and (58), we have that

$$
\left|w_{n}\right| \leq M \int_{0}^{R} G(r, s) s^{N-1}\left|w_{n}\right| \mathrm{d} r
$$

where $M=\max \left\{\alpha^{0}, \beta^{0}\right\}$. By the Gronwall-Bellman inequality ([23, Lemma 2.1]), we obtain that $\|w\|=0$. This contradicts the fact of $\|w\|=1$. Hence, we have that $\mathscr{D}^{\nu} \subset\left(\mathbb{R} \times P^{\nu}\right)$.

Finally, we show that $\operatorname{Proj}_{\mathbb{R}}\left(\mathscr{D}_{k}^{v}\right) \neq \varnothing$. From the argument of Theorem 3, we have known that $\mathscr{D}_{k}^{v[n]}$ has unbounded projection on $\mathbb{R}$ for any fixed $n \in \mathbb{N}$. By Lemma 7 , for each fixed $\epsilon>0$ there exists an $m$ such that, for every $n>m, \mathscr{D}_{k}^{v[n]} \subset V_{\epsilon}\left(\mathscr{D}_{k}^{v}\right)$. This implies that

$$
\left(\lambda^{\nu} n,+\infty\right) \subseteq \operatorname{Proj}_{\mathbb{R}}\left(\mathscr{D}_{k}^{\nu[n]}\right) \subseteq \operatorname{Proj}_{\mathbb{R}}\left(V_{\varepsilon}\left(\mathscr{D}_{k}^{\nu}\right)\right),
$$


where $\operatorname{Proj}_{\mathbb{R}}\left(\mathscr{D}_{k}^{v}\right)$ denotes the projection of $\mathscr{D}_{k}^{v}$ on $\mathbb{R}$. It follows that the projection of $\mathscr{D}_{k}^{v}$ is nonempty on $\mathbb{R}$.

By an argument similar to that of Theorem 4, we can get the asymptotic behavior of $\lim _{k \longrightarrow \infty}\|u\|=0$ for $(\lambda, u) \in \mathscr{D}_{k}^{v}$.

\section{Data Availability}

Data sharing not applicable to this paper as no datasets were generated or analyzed during the current study.

\section{Conflicts of Interest}

The authors declare that they have no conflicts of interest.

\section{Authors' Contributions}

The author conceived the study, drafted the manuscript, and approved the final manuscript.

\section{Acknowledgments}

This research was supported by the NSFC (no. 11561038) and "Kaiwu"Innovation Team Support Project of Lanzhou Institute of Technology (no. 2018KW-03).

\section{References}

[1] L. J. Alías and B. Palmer, "On the Gaussian curvature of maximal surfaces and the Calabi-Bernstein theorem," Bulletin of the London Mathematical Society, vol. 33, no. 4, pp. 454458, 2001.

[2] C. Bereanu, P. Jebelean, and J. Mawhin, "Multiple solutions for Neumann and periodic problems with singular $\phi$-Laplacian," Journal of Functional Analysis, vol. 261, no. 11, pp. 3226-3246, 2011.

[3] C. Bereanu, P. Jebelean, and J. Mawhin, "Radial solutions of Neumann problems involving mean extrinsic curvature and periodic nonlinearities," Calculus of Variations and Partial Differential Equations, vol. 46, no. 1-2, pp. 113-122, 2013.

[4] S.-Y. Cheng and S.-T. Yau, "Maximal space-like hypersurfaces in the Lorentz-Minkowski spaces," The Annals of Mathematics, vol. 104, no. 3, pp. 407-419, 1976.

[5] A. E. Treibergs, "Entire spacelike hypersurfaces of constant mean curvature in Minkowski space," Inventiones Mathematicae, vol. 66, no. 1, pp. 39-56, 1982.

[6] M. F. Bidaut-Véron and A. Ratto, "Spacelike graphs with prescribed mean curvature," Differential and Integral Equations, vol. 10, no. 5, pp. 1003-1017, 1997.

[7] R. López, "Stationary surfaces in Lorentz-Minkowski space," Proceedings of the Royal Society of Edinburgh: Section A Mathematics, vol. 138, no. 5, pp. 1067-1096, 2008.

[8] C. Bereanu, P. Jebelean, and P. J. Torres, "Positive radial solutions for Dirichlet problems with mean curvature operators in Minkowski space," Journal of Functional Analysis, vol. 264, no. 1, pp. 270-287, 2013.

[9] C. Bereanu, P. Jebelean, and P. J. Torres, "Multiple positive radial solutions for a Dirichlet problem involving the mean curvature operator in Minkowski space," Journal of Functional Analysis, vol. 265, no. 4, pp. 644-659, 2013.

[10] R. Ma, H. Gao, and Y. Lu, "Global structure of radial positive solutions for aprescribed mean curvature problem in a ball,"
Journal of Functional Analysis, vol. 270, no. 7, pp. 2430-2455, 2016.

[11] G. Dai, "Bifurcation and positive solutions for problem with mean curvature operator in Minkowski space," Calculus of Variations and Partial Differential Equations, vol. 55, no. 4, p. 72, 2016.

[12] G. Dai and J. Wang, "Nodal solutions to problem with mean curvature operator in Minkowski space," Differential and Integral Equations, vol. 30, no. 5-6, pp. 463-480, 2017.

[13] H. Berestycki, "On some nonlinear Sturm-Liouville problems," Journal of Differential Equations, vol. 26, no. 3, pp. 375-390, 1977.

[14] G. Dai and R. Ma, "Unilateral global bifurcation for $p$-Laplacian with non- $p$ - 1-lineariza-tion nonlinearity," Discrete \& Continuous Dynamical Systems-A, vol. 35, no. 1, pp. 99-116, 2015.

[15] G. Dai and R. Ma, "Global bifurcation, Berestycki's conjecture and one-sign solutions for $p$-Laplacian," Nonlinear Analysis: Theory, Methods \& Applications, vol. 91, pp. 51-59, 2013.

[16] G. Dai and B. Yang, "Positive answer to Berestyckis open problem on the unit ball," Advanced Nonlinear Studies, vol. 16, no. 4, pp. 643-652, 2016.

[17] G. Dai, R. Ma, and J. Xu, "Global bifurcation and nodal solutions of $\mathrm{N}$-dimensional $p$-Laplacian in unit ball," Applicable Analysis, vol. 92, no. 7, pp. 1345-1356, 2013.

[18] P. H. Rabinowitz, "Some global results for nonlinear eigenvalue problems," Journal of Functional Analysis, vol. 7, no. 3, pp. 487-513, 1971.

[19] A. Capietto, W. Dambrosio, and F. Zanolin, "Infinitely many radial solutions to a boundary value problem in a ball," Annali di Matematica Pura ed Applicata, vol. 179, no. 1, pp. 159-188, 2001.

[20] G. Dai, "Bifurcation and one-sign solutions of the $p$-Laplacian involving a nonlinearity with zeros," Discrete and Continuous Dynamical Systems, vol. 36, no. 10, pp. 5323-5345, 2016.

[21] G. T. Whyburn, Topological Analysis, Princeton University Press, Princeton, NJ, USA, 1958.

[22] A. Ambrosetti, R. M. Calahorrano, and F. R. Dobarro, "Global branching for discontinuous problems," Commentationes Mathematicae Universitatis Carolinae, vol. 31, pp. 213-222, 1990.

[23] B. H. Im, E. K. Lee, and Y.-H. Lee, “A global bifurcation phenomenon for second order singular boundary value problems," Journal of Mathematical Analysis and Applications, vol. 308, no. 1, pp. 61-78, 2005. 